\title{
Crohn's Disease Presenting as Ileo-Ileal Intussusception: A Case Report
}

\section{Maral Rouhani ${ }^{1}$, Mohammed Rashid ${ }^{2 *}$, Boby Sebastian ${ }^{2}$, Timothy Justin ${ }^{2}$}

1 University of Cambridge, The Old Schools, Trinity Lane, Cambridge, United Kingdom

${ }^{2}$ Department of General Surgery, West Suffolk Hospital, Bury St Edmunds, Suffolk, United Kingdom

\begin{abstract}
The authors present an unusual case of a 44 year old man who initially presented with urinary symptoms and underwent a CT scan of his abdomen, which showed an incidental finding of terminal ileal intussusception, which was investigated and found to be Crohn's related.
\end{abstract}

Keywords: Ileal intussusception crohn's

\section{Introduction}

Crohn's disease is a chronic inflammatory condition affecting the bowel [1], the incidence of which is ever increasing in Europe and the USA [2]. Diarrhoea, abdominal pain and weight loss are common presenting symptoms [3], but to date, there have been no cases in the literature of it being diagnosed after an incidental finding of a small bowel intussusception on a CT scan. Here, the authors present such as case.

\section{Case Report}

A 44 year old man was initially referred to the urology clinic in June 2011 with urinary symptoms including left sided loin pain and hesitancy. His past medical history included hypertension, asthma and varicose veins. He was a nonsmoker and only a social drinker. There was no family history of bowel problems. He had a renal ultrasound scan which showed a mild left sided hydronephrosis and was investigated further with a CT of his abdomen and pelvis. This showed a $13 \mathrm{~mm}$ ureteric calculus (Figure 1) in the left proximal ureter causing mild hydronephrosis and also an ileo-ileal intussusception, a few small adjacent mesenteric nodes and mesenteric thickening (Figures 2, 3). He underwent a flexible ureteroscopy and lithotripsy in August 2011, after which all his symptoms resolved. In view of the intussusception he was reviewed in the surgical clinic, where it was noted that he was asymptomatic. Physical examination was unremarkable as were routine blood tests. He was investigated further with colonoscopy, which revealed erythema, granularity and aphthous ulcers within the colon and terminal ileum (Figure 4). Biopsies were consistent with inflammatory bowel disease of Crohn's type, with no dysplasia. A barium follow through in November 2011 showed evidence of terminal

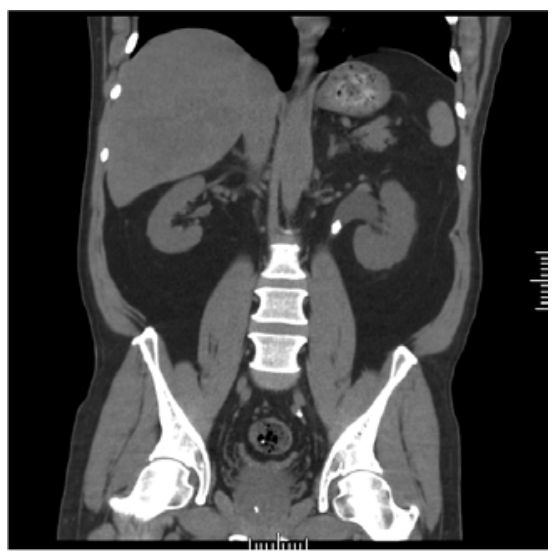

Figure 1: Left ureretic stone on a coronal CT view.

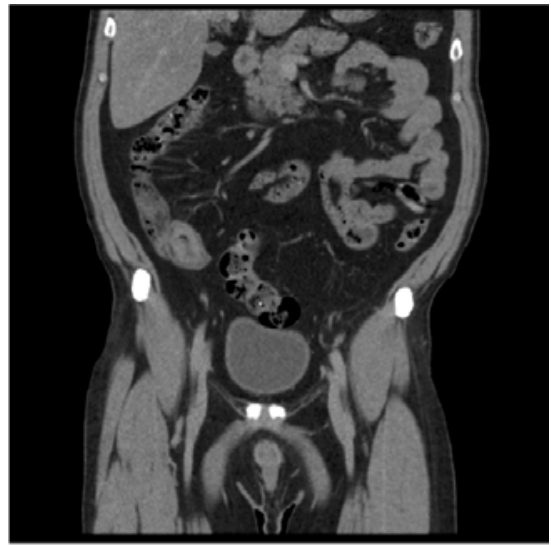

Figure 2: Ileal-lleal Intussusception on a Coronal CT View.

ileal inflammation, with two areas of mild structuring, with resolution of the intussusception (Figure 5).

\section{Discussion}

Crohn's disease is a chronic granulomatous inflammatory disease that may involve any part of the bowel, although the terminal ileum is the most frequently affected location. The presenting symptoms are variable and include abdominal pain, diarrhoea and weight loss. The incidence has been reported to vary worldwide, from 0.7 (Croatia) to 15.6 (Canada) new cases per 100,000 people per year [2]. Internationally accepted diagnostic criteria are defined by Lennard-Jones and include the presence of chronic inflammatory lesions in the bowel, a discontinuous disease distribution, transmural inflammation, fibrosis, lymphoid aggregates or aphthoid ulcers and retention of colonic mucin on biopsy in the presence of active inflammation [4].

Intussusception occurs when a proximal section of bowel invaginates into another adjacent section. It is more common in young children, where causes include any lesion in the lumen or disorganised peristalsis [5]. Only around 5\% of all cases of intussusception occur in

*Corresponding author: Mohammed Rashid, Department of General Surgery, West Suffolk Hospital, Suffolk, E-mail: amrd02@gmail.com

Received March 22, 2012; Accepted April 19, 2012; Published April 20, 2012

Citation: Rouhani M, Rashid M, Sebastian B, Justin T (2012) Crohn's Disease Presenting as Ileo-lleal Intussusception: A Case Report. Surgery Curr Res 2:114 doi:10.4172/2161-1076.1000114

Copyright: (c) 2012 Rouhani M, et al. This is an open-access article distributed under the terms of the Creative Commons Attribution License, which permits unrestricted use, distribution, and reproduction in any medium, provided the original author and source are credited. 


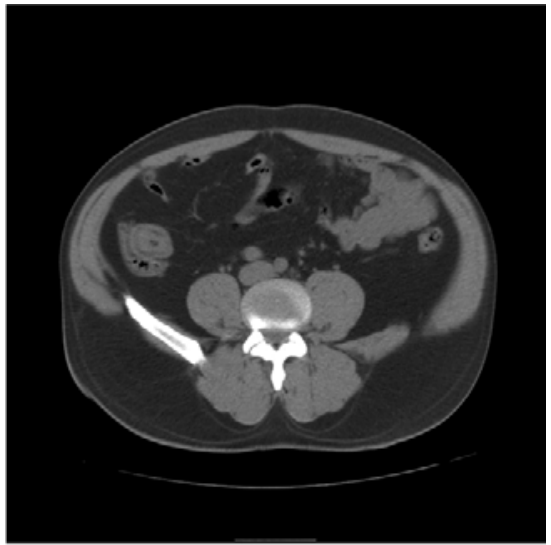

Figure 3: lleal-lleal Intussusception on an Axial CT View.

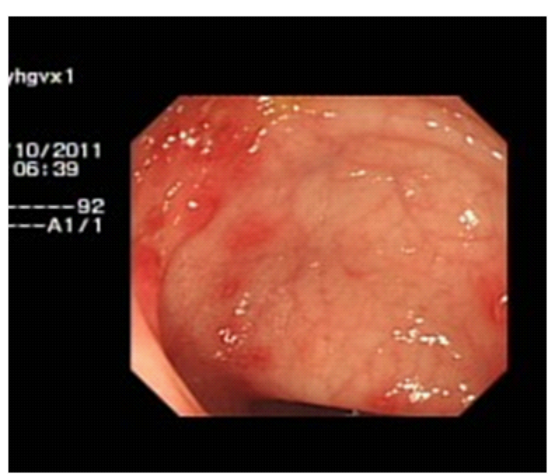

Figure 4: A photo of the proximal sigmoid colon showing patchy areas of mild inflammation and apthous ulcers.

adults [6]. Of these, $80-90 \%$ is secondary to an underlying pathology such as a tumour.

Crohn's disease presenting as an asymptomatic intussusception is rare in the literature. One such case in 2006, describes a patient who presented with an acute abdomen secondary to an intussusception from Crohn's disease but in this case, intestinal necrosis secondary to the ileoileal intussusception necessitated a small bowel resection, after which the patient made an uneventful recovery [7]. Also, transient intussusception has been reported before. A report from 1989 of two

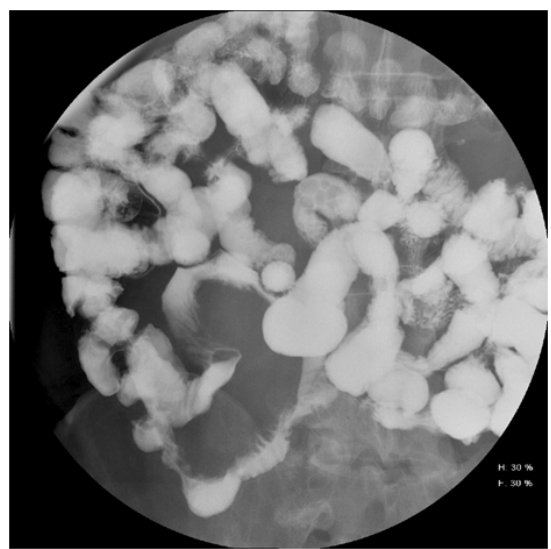

Figure 5: A barium follow through showing inflammation of the terminal ileum.

patients who were found to have a Crohn's related intussusception demonstrated on CT scan initially, found that later on, both intussusceptions had resolved on a repeat CT [8], without treatment.

\section{Conclusion}

Consideration should be given to Crohn's disease as a cause, in younger patients with intestinal intussusception.

\section{References}

1. Agha FP (1986) Intussusception in adults. AJR Am J Roentgenol 146: 527-531.

2. López-Tomassetti Fernández EM, Lorenzo Rocha N, Arteaga González I Carrillo Pallarés A (2006) lleoileal intussusception as initial manifestation of Crohn's disease. Mcgill J Med 9: 34-37.

3. Knowles MC, Fishman EK, Kuhlman JE, et al. (1989) Transient intussusception in Crohn's disease: CT evaluation. Radiology 170:814.

4. Lennard-Jones JE (1989) Classification of inflammatory bowel disease Scandinavian Journal of Gastroenterology 170: 2-6.

5. Milbrandt K, Sigalet D (2008) Intussusception associated with a Meckel's diverticulum and a duplication cyst. J Pediatr Surg. 43: e21-e23.

6. Jewell DP. Crohn's disease. In: Warrell DA, Cox TM, Firth JD, et al, eds. Oxford textbook of medicine. 4th ed. Oxford: Oxford University Press, 2005.

7. Loftus EV Jr. (2004) Clinical epidemiology of inflammatory bowel disease incidence, prevalence, and environmental influences. Gastroenterology 126:1504-1517.

8. Stange EF, Travis SP, Vermeire S, C Beglinger4, L Kupcinkas, et al. (2006) European evidence based consensus on the diagnosis and management of Crohn's disease: definitions and diagnosis. Gut 55: i1-i15. 